\title{
Overexpression of Canonical Prefoldin Associates with the Risk of Mortality and Metastasis in Non-Small Cell Lung Cancer
}

\author{
Xenia Peñate ${ }^{1}$, Juan Manuel Praena-Fernández ${ }^{2}$, , Pedro Romero Pareja ${ }^{3}$, \\ María del Valle Enguix-Riego ${ }^{3}$, Laura Payán-Bravo ${ }^{1}$, Begoña Vieites ${ }^{4}$, \\ Lourdes Gomez-Izquierdo ${ }^{4}\left(\mathbb{D}\right.$, Javier Jaen Olasolo ${ }^{5}$, Eleonor Rivin del Campo ${ }^{6} \mathbb{D}$, \\ Jose Carlos Reyes ${ }^{7}$, Sebastián Chávez ${ }^{1, *(\mathbb{D})}$ and Jose Luis Lopez Guerra ${ }^{3, *}$ (D) \\ 1 Instituto de Biomedicina de Sevilla, Universidad de Sevilla-CSIC-Hospital Universitario V. del Rocío, \\ Avda. Manuel Siurot s/n, 41013 Seville, Spain; xenia@us.es (X.P.); laupaybra@gmail.com (L.P.-B.) \\ 2 Department of Nursing, Universidad de Sevilla, 41009 Seville, Spain; jmpraenaf@gmail.com \\ 3 Department of Radiation Oncology, University Hospital Virgen del Rocío, Avda. Manuel Siurot s/n, \\ 41013 Seville, Spain; pedro.romero.pareja@gmail.com (P.R.P.); enguixriego@gmail.com (M.d.V.E.-R.) \\ 4 Department of Pathology, University Hospital Virgen del Rocío, Avda. Manuel Siurot s/n, 41013 Seville, \\ Spain; b.vieites.pq@gmail.com (B.V.); gomezizquierdo.l@gmail.com (L.G.-I.) \\ 5 Department of Radiation Oncology, University Hospital Puerta del Mar, Av. Ana de Viya, 21, 11009 Cadiz, \\ Spain; jjaen2008@gmail.com \\ 6 Department of Radiation Oncology, Tenon University Hospital, Sorbonne University, 4 Rue de la Chine, \\ 75020 Paris, France; eleonorrivin@gmail.com \\ 7 Andalusian Center of Molecular Biology and Regenerative Medicine-CABIMER, \\ Junta de Andalucia-University of Pablo de Olavide-University of Seville-CSIC, 41092 Seville, Spain; \\ jose.reyes@cabimer.es \\ * Correspondence: schavez@us.es (S.C.); chanodetriana@yahoo.es (J.L.L.G.); \\ Tel.: +34-95-501-2105 (S.C. \& J.L.L.G.); Fax: +34-95-501-2111 (S.C. \& J.L.L.G.)
}

Received: 27 March 2020; Accepted: 20 April 2020; Published: 24 April 2020

Abstract: Canonical prefoldin is a protein cochaperone composed of six different subunits (PFDN1 to 6). PFDN1 overexpression promotes epithelial-mesenchymal transition (EMT) and increases the growth of xenograft lung cancer (LC) cell lines. We investigated whether this putative involvement of canonical PFDN in LC translates into the clinic. First, the mRNA expression of 518 non-small cell LC (NSCLC) cases from The Cancer Genome Atlas (TCGA) database was evaluated. Patients with PFDN1 overexpression had lower overall survival (OS; 45 vs. 86 months; $p=0.034)$. We then assessed the impact of PFDN expression on outcome in 58 NSCLC patients with available tumor tissue samples. PFDN1, 3, and 5 overexpression were found in 38\% $(n=22), 53 \%(n=31)$, and $41 \%(n=24)$ of tumor samples. PFDN1, 3, and 5 overexpression were significantly associated with lower OS, lower disease-free survival (DFS), and lower distant metastasis-free survival (DMFS) for PFDN1 and 3 with a trend for PFDN5. In multivariate analysis, PFDN5 retained significance for OS (hazard ratio (HR) $2.56 ; p=0.007$ ) and PFDN1 for DFS (HR 2.53; $p=0.010$ ) and marginally for DMFS (HR 2.32; $p=0.053$ ). Our results indicate that protein response markers, such as PFDN1, 3, and 5, may complement mRNA signatures and be useful for determining the most appropriate therapy for NSCLC patients.

Keywords: prefoldin; metastasis; survival; non-small cell lung cancer

\section{Introduction}

The high incidence of metastasis is one of the main components of mortality in non-small cell lung cancer (NSCLC). Cancer epithelial cells need to transform into mesenchymal cells to escape the 
primary tumor and circulate towards the target tissues where secondary tumors are established $[1,2]$. Epithelial-mesenchymal transition (EMT) results in the loss of epithelial markers and the increase in mesenchymal markers. During EMT, cells acquire partial pluripotency and stemness [3], particularly during intermediate states [4].

EMT is driven by immune cells and inflammation in the microenvironment of lung tumors [5], which activate several signaling pathways with a predominant role of the transforming growth factor- $\beta$ (TGF- $\$$ ) family of cytokines [6]. EMT in cancer is also associated with the repression of genes that promote cell cycling [7]. This repression seems to be promoted by the accumulation of prefoldin 1 (PFDN1), in response to TGF- $\beta 1$-mediated activation [8]. PFDN1 is a subunit of prefoldin, a cochaperone that contributes to protein folding in cooperation with type II adenosine triphosphate-dependent chaperonins [9]. Canonical prefoldin shows a jellyfish-like structure and is composed of six different subunits (PFDN1 to 6). PFDNs also participate in processes that take place in the cell nucleus [10], including molecular events that contribute to gene regulation [11].

The involvement of PFDN1 in promoting EMT in lung cancer suggests that PFDN accumulation may play an important role in the evolution of lung cancer and the onset of metastasis. However, the available evidence only comes from in vitro work with cell lines and mice xenografts [8]. We report the results of a translational study that show the potential utility of PFDN in predicting overall survival (OS) and metastasis.

\section{Methods and Materials}

\section{1. mRNA Expression}

The mRNA expression (RNA-seq) and clinical data of 518 NSCLC cases from The Cancer Genome Atlas (TCGA) database (http://cancergenome.nih.gov/) were evaluated. First, the survival plots of patients with tumors that expressed low vs. high levels of $P F D N$ were analyzed. We then compared the levels of PFDN gene transcripts in normal tissue $(n=59)$ and the tumor tissue $(n=517)$.

\subsection{Patient Population}

Clinical data from 58 NSCLC patients (Table 1) were collected prospectively between 2001 and 2017 and were used for this analysis. There were no notable differences in the type of chemotherapy and radiotherapy used. First-line systemic therapy consisted of platinum-based double-agent chemotherapy in all cases except one who received a protein kinase inhibitor (afatinib). A three-dimensional conformal radiotherapy technique was used in all cases except one who was treated with a volumetric arc therapy technique. Only 3 patients had epidermal growth factor receptor (EGFR) mutations, and there were not any anaplastic lymphoma kinase (ALK) translocations. In addition, only 3 patients were programmed cell death-ligand 1 (PD-L1) positive. Exclusion criteria included having either small cell lung cancer histology or previous oncologic treatments.

Table 1. Patient characteristics.

\begin{tabular}{lc}
\hline \multicolumn{1}{c}{ Characteristics } & $\begin{array}{c}\text { NO. of Patients (\%) } \\
\boldsymbol{n}=58\end{array}$ \\
\hline Gender & \\
$\quad$ Female & $8(14)$ \\
Male & $50(86)$ \\
\hline Age, years & \\
$\quad$ Median (range) & $67(41-82)$ \\
\hline Chronic obstructive pulmonary disease & \\
$\quad$ No & $34(59)$ \\
Yes & $24(41)$ \\
\hline Hypertension & \\
No & $31(53)$ \\
Yes & $27(57)$ \\
\hline
\end{tabular}


Table 1. Cont.

\begin{tabular}{|c|c|}
\hline Characteristics & $\begin{array}{c}\text { NO. of Patients }(\%) \\
n=58\end{array}$ \\
\hline \multicolumn{2}{|l|}{ Diabetes Mellitus } \\
\hline No & $42(58)$ \\
\hline Yes & $16(42)$ \\
\hline \multicolumn{2}{|l|}{ Dyslipidemia } \\
\hline No & $26(45)$ \\
\hline Yes & $32(55)$ \\
\hline \multicolumn{2}{|l|}{ Cardiovascular disease } \\
\hline No & $45(78)$ \\
\hline Yes & $13(22)$ \\
\hline \multicolumn{2}{|l|}{ Thrombosis } \\
\hline No & $49(84)$ \\
\hline Yes & $9(16)$ \\
\hline \multicolumn{2}{|l|}{ Smoking status } \\
\hline Never & $3(5)$ \\
\hline Former & $28(48)$ \\
\hline Current & $27(47)$ \\
\hline \multicolumn{2}{|c|}{ History of alcohol consumption } \\
\hline No & $31(53)$ \\
\hline Yes & $27(47)$ \\
\hline \multicolumn{2}{|c|}{ Karnofsky Performance Status } \\
\hline 100 & $13(22)$ \\
\hline 90 & $15(26)$ \\
\hline 80 & $16(28)$ \\
\hline 70 & $14(24)$ \\
\hline \multicolumn{2}{|l|}{ Histology } \\
\hline Adenocarcinoma & $24(41)$ \\
\hline Squamous & $34(59)$ \\
\hline \multicolumn{2}{|l|}{ T stage } \\
\hline $\mathrm{T} 1$ & $13(22)$ \\
\hline $\mathrm{T} 2$ & $28(48)$ \\
\hline T3 & $11(19)$ \\
\hline $\mathrm{T} 4$ & $6(10)$ \\
\hline \multicolumn{2}{|l|}{$\mathrm{N}$ stage } \\
\hline N0 & $19(33)$ \\
\hline N1 & $8(14)$ \\
\hline $\mathrm{N} 2$ & $25(43)$ \\
\hline N3 & $6(10)$ \\
\hline \multicolumn{2}{|l|}{ M stage } \\
\hline M0 & $53(91)$ \\
\hline M1 & $5(9)$ \\
\hline \multicolumn{2}{|l|}{ Stage } \\
\hline IA & $1(2)$ \\
\hline IB & $13(22)$ \\
\hline IIA & $4(7)$ \\
\hline IIIA & $27(47)$ \\
\hline IIIB & $8(14)$ \\
\hline IV & $5(9)$ \\
\hline \multicolumn{2}{|l|}{ Surgery } \\
\hline Yes & $52(90)$ \\
\hline No & $6(10)$ \\
\hline \multicolumn{2}{|c|}{ Thoracic radiation therapy * } \\
\hline Yes & $24(41)$ \\
\hline No & $34(59)$ \\
\hline \multicolumn{2}{|l|}{ Chemotherapy ** } \\
\hline Yes & $40(69)$ \\
\hline No & $18(31)$ \\
\hline
\end{tabular}

${ }^{*}$ Delivery with radical intent; ${ }^{* *}$ Delivery with radical intent in all cases except one. 


\subsection{Tissue Microarrays Immunohistochemical Analysis}

Immunohistochemical studies were performed on lung cancer specimens in a tissue microarray (TMA). The tumor samples were obtained from our Institutional Biobank and were stored in paraffin blocks of lung carcinoma. Two independent pathologists, blinded to patient data, performed tissue sampling and scored PFDN expression. Antibodies were obtained from commercially available sources: Anti-PFDN1 (ab151708) and Anti-PFDN3 (ab96085) antibodies (Abcam, Cambridge, UK); Anti-PFDN5 (sc-27119) (Santa Cruz Biotechnology, Dallas, TX, USA). The following discrete values were assigned for observations: 0 , no expression; $1(+)$, weak expression; $2(++)$, strong expression, and $3(+++)$, very strong expression (Figure S1).

\subsection{Statistical Analysis}

SPSS (version 26.0, IBM Corp., Armonk, NY, USA) statistical software and GraphPad Prism version 5.0 (GraphPad, San Diego, CA, USA) were used for data analyses. The primary outcome was OS. The Kaplan-Meier method provided estimates of the following endpoints: OS, disease-free survival [DFS; defined as any disease recurrence (loco-regional, or distant)], loco-regional recurrence (LR), and distant metastases (DM). Multivariate analyses, including the statistically significant features in the univariate analysis, were performed using Cox's proportional hazard model. $p \leq 0.05$ was considered significant.

\section{Results}

\subsection{PFDN1 mRNA Levels Associates with OS in NSCLC}

We first analyzed the TCGA lung cohort and found that patients with high-PFDN1 tumors had a median survival of 45 months, while patients with low-PFDN1 tumors presented a median survival of 86 months (log-rank test $p$-value $=0.034$; Figure 1A). No significant differences in OS of patients with low-PFDN versus high-PFDN were found for the other five PFDN genes (Figure 1A).

We also found that levels of PFDN1-6 transcripts were significantly higher in tumors with high PFDNs levels than in normal tissue (Figure 1B). There were no significant differences in EGFR mutations among patients with high and low levels of PFDN1 $(p=0.896), 2(p=0.549), 3(p=0.938), 4(p=0.735)$, $5(p=0.618)$, and $6(p=0.735)$ mRNA. ALK translocations were significantly lower only in patients with higher levels of PFDN4 mRNA (39\% vs. $61 \%$; $p=0.034$ ).

There was a high correlation between PFDN2 and 4 mRNA levels in tumor samples (Spearman coefficient: $0.580 ; p<0.0001$ ) and between PFDN2 and 6 (Spearman coefficient: $0.640 ; p<0.0001$; Figure 1C). In contrast, levels of PFDN1 and PFDN5 transcripts, which also correlated with each other (Spearman coefficient: 0.53; $p<0.0001$; Figure 1C), were either invariant or increased in normal tissue with respect to tumors (Figure 1B). This discrepancy prompted us to analyze PFDN protein levels in tumor samples by immunohistochemistry. 


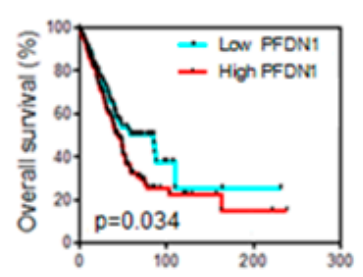

Time (Months)
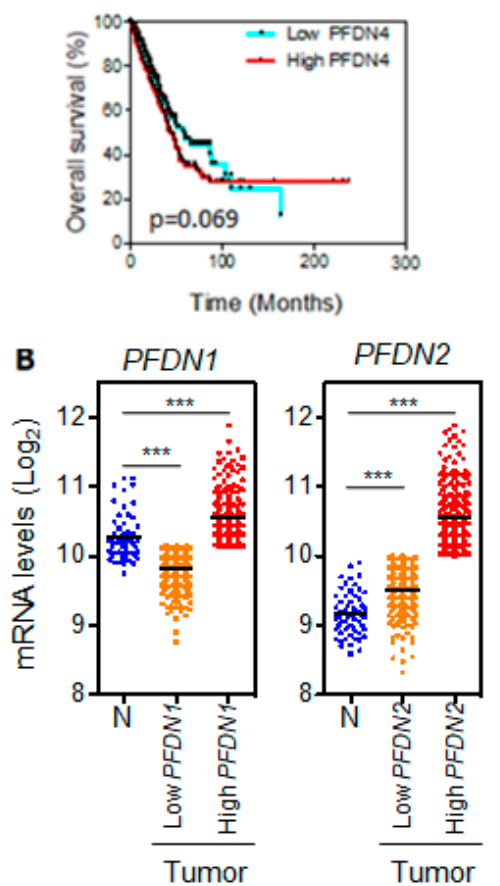

C

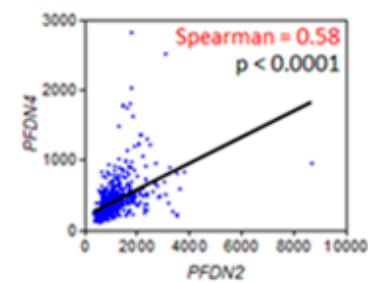

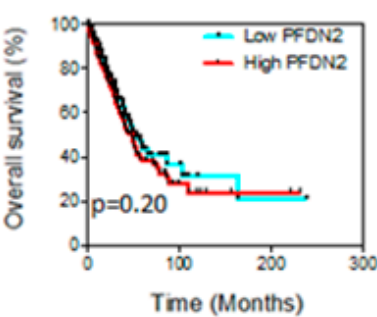
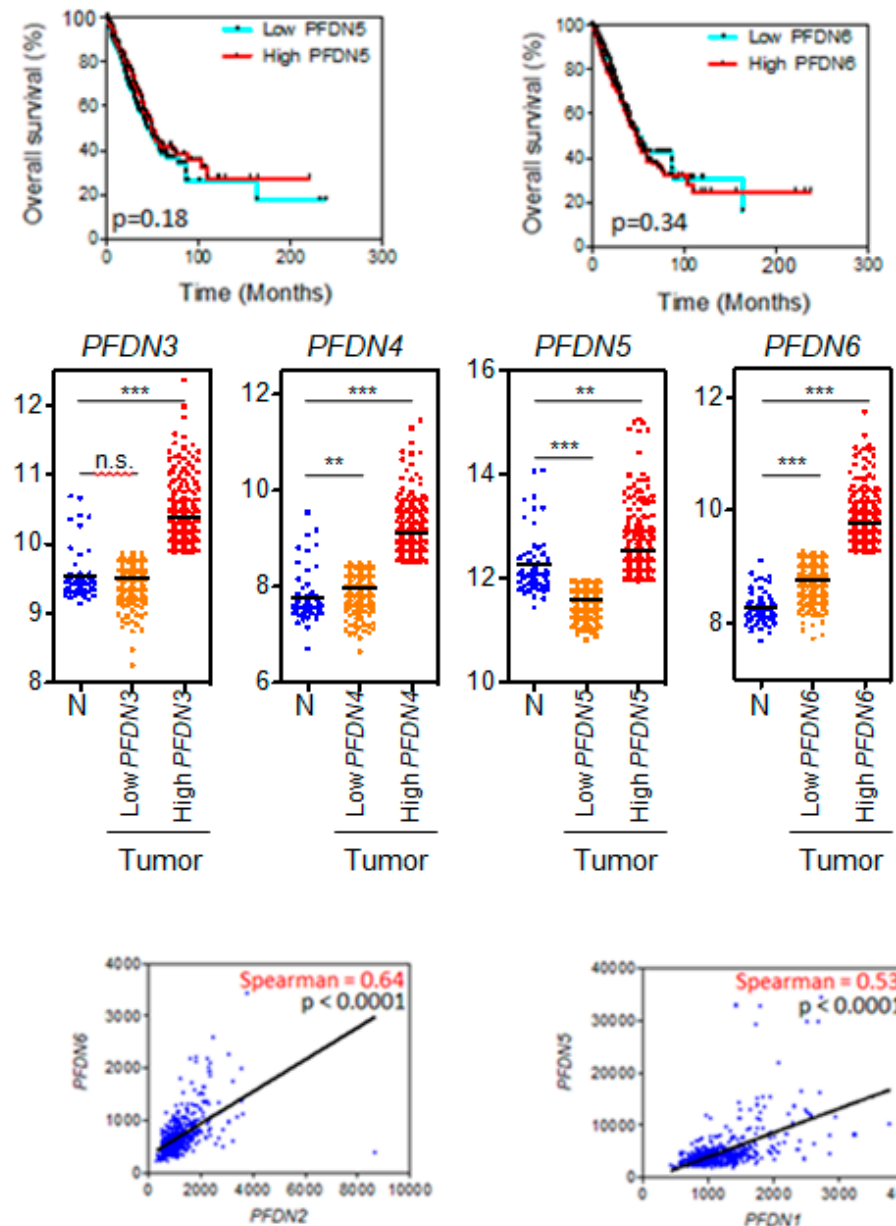
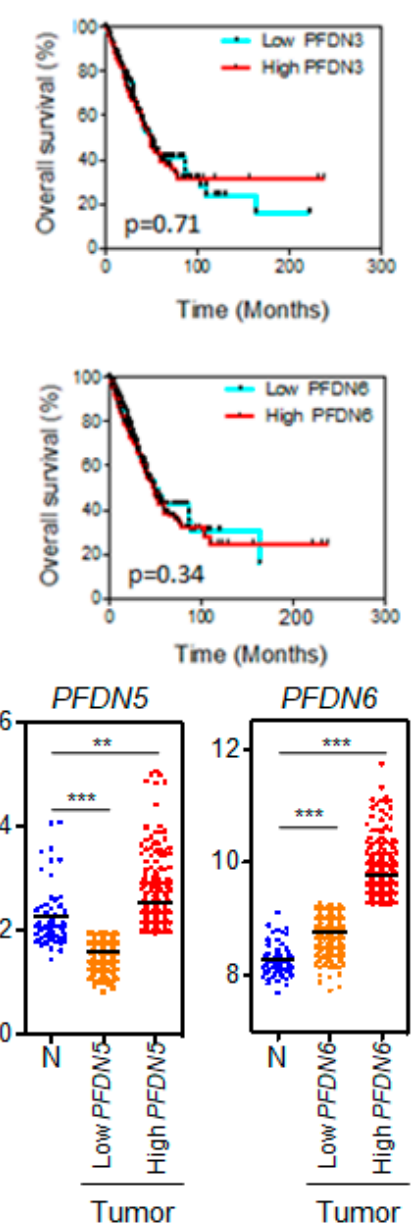

Time (Months)

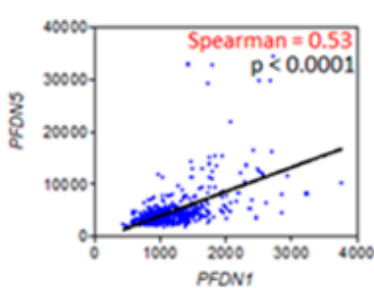

Figure 1. (A) Kaplan-Meier curve of The Cancer Genome Atlas (TCGA) lung cohort for overall survival according to low vs. high expression of prefoldins (PFDNs) (cut off point: 50\%); (B) Levels of PFDNs transcripts in tumors and normal tissue (Abbreviations: $\mathrm{N}$, normal; n.s., not significant; *** $p<0.0001 ; * * 0.001)$. Tumor samples were divided into two categories: low and high expression of PFDNs as in (A); (C) Significant correlations between mRNA levels of different PFDN genes in tumor samples. RNA-seq expression data are provided as RNA-Seq by Expectation Maximization (RSEM) normalized data.

\subsection{Association of PFDN and Survival in NSCLC Patients}

The clinical and pathological characteristics of the patients enrolled in the study are summarized in Table 1. Median follow-up time for all patients was 40 months (range, 5 to 168 months). At the time of analysis, $67 \%(n=39)$ were alive, 33\% $(n=19)$ had died, and $58 \%(n=34)$ had experienced a recurrence ( 24 were distant metastasis).

PFDN1, 3, and 5 overexpression $(+++)$ was found in $38 \%(n=22), 53 \%(n=31)$, and $41 \%(n=24)$ of tumor samples. There was a high correlation among PFDNs levels $(p<0.010$; Figure 2A). There were no significant differences in the stage distribution among patients with and without PFDN1, 3 , 
and 5 overexpression ( $\mathrm{p} 0.098, p=0.724$, and $p=0.796$, respectively). There were $4(18 \%)$ and $18(82 \%)$ patients with early (I/II) and advanced (III/IV) stages, respectively, in the PFDN1 overexpressed cohort, and $14(39 \%)$ and $22(61 \%)$ patients with early and advanced stages, respectively, in the PFDN1 cohort without overexpression. There were $9(29 \%)$ and $22(71 \%)$ patients with early and advanced stages, respectively, in the PFDN3 overexpressed cohort, and $9(33 \%)$ and $18(67 \%)$ patients with early and advanced stages, respectively, in the PFDN3 cohort without overexpression. There were $7(29 \%)$ and $17(71 \%)$ patients with early and advanced stages, respectively, in the PFDN5 overexpressed cohort, and $11(32 \%)$ and $23(68 \%)$ patients with early and advanced stages, respectively, in the PFDN5 cohort without overexpression. Associations between corresponding patient's clinic-pathological features and OS, DFS, local and distant relapse are shown in Table S1.
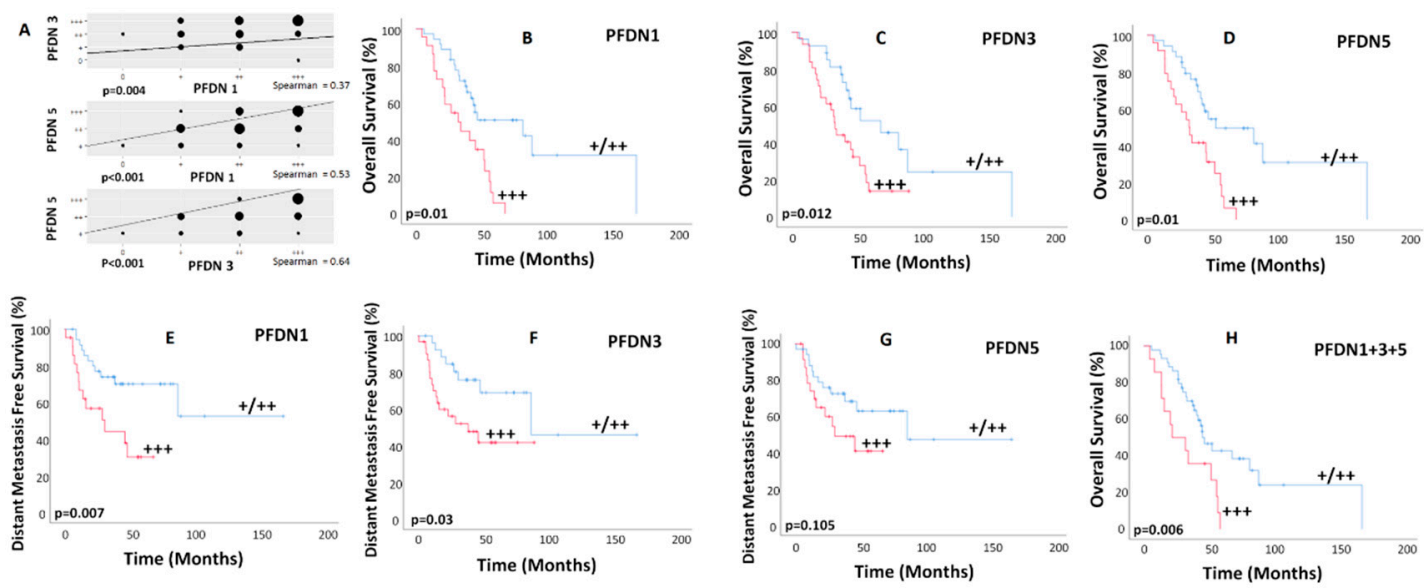

Figure 2. (A) Correlation among PFDN protein levels for all 58 patients. The diameter of the dots is proportional to the number of cases. (B-H) Kaplan-Meier curves for overall survival (B-D) and distant metastasis-free survival (E-G) according to PFDN expression and overall survival when combining all overexpressed PFDNs $(\mathbf{H})$.

PFDN1, 3, and 5 overexpression were associated with lower OS (HR: 2.86, CI 1.47-5.56, $p=0.002$; HR: 2.30, CI 1.17-4.50, $p=0.015$; and HR: 2.94, CI 1.50-5.76, $p=0.002$, respectively; Figure 2B-D), lower DFS (HR: 2.53, CI 1.24-5.15, $p=0.010$; HR: 2.09, CI 1.02-4.27, $p=0.042$; and HR: 2.002, CI 0.986-4.064, $p=0.055$, respectively; Figure S2), and lower distant metastasis-free survival (DMFS) for PFDN1 and 3 with a trend for PFDN5 (HR: 2.94, CI 1.28-6.73, $p=0.011$; HR: 2.50, CI 1.06-5.88, $p=0.036$; and HR: 1.94, CI $0.85-4.42, p=0.112$, respectively; Figure $2 \mathrm{E}-\mathrm{G}$ ). The majority of patients ( $n=48 ; 83 \%$ ) were treated between 2012 and 2017. Similar results were found for this more recent cohort. Patients with PFDN1, 3 , and 5 overexpression showed significantly lower overall survival $(p=0.011, p=0.046$, and $p=0.013$, respectively). In addition, those with PFDN1 and 3 overexpression had also lower disease-free survival ( $p=0.023$ and $p=0.042$, respectively) and distant metastasis-free survival ( $p=0.013$ and $p=0.046$, respectively). Additionally, there were no significant differences $(p>0.05)$ in the type of surgery as well as in the use of radiotherapy or chemotherapy among patients with and without PFDN1, 3, and 5 overexpression.

There was no association with local recurrence. In multivariate analysis (Table S2), PFDN5 retained significance for OS (HR 3.13; CI 1.59-6.15, $p=0.001$ ) and PFDN1 for DFS (HR 2.53; CI 1.24-5.15, $p=0.010$ ) and DMFS (HR 2.32; CI 0.98-5.45, $p=0.053$; Table S2).

When combining overexpressed PFDNs, Kaplan-Meier curves for mortality estimated OS at 24 months for $50 \%$ of patients with PFDN1, 3, and 5 overexpression (HR: 2.550; CI: 1.280-5.090; $p=0.008$; Figure $2 \mathrm{H}$ ) and $86 \%$ for the rest of patients. Two-year DFS rate for patients with PFDN1 and 3 overexpression was $35 \%$ vs. $70 \%$ for those with lower expression (HR: 2.370; CI: 1.140-4.900; $p=0.020$; Figure S3A). In addition, patients with PFDN1 and 3 overexpression had a 2-year DMFS rate 
of $49 \%$ vs. $78 \%$ in the subset of patients with lower expression (HR: 2.880 ; CI: $1.250-6.600 ; p=0.012$ : Figure S3B).

\section{Discussion}

Overexpression of canonical PFDN associates with the risk of mortality and metastasis in non-small cell lung cancer (LC). Our pertinent findings indicate that patients with PFDN5 overexpression in the tumor tissue had higher mortality rates, and those having PFDN1 overexpression also showed higher rates of recurrence, specifically for distant metastasis. When combining overexpressed PFDNs, patients with PFDN1, 3, and 5 overexpression had lower OS, and those with PFDN1 and 3 overexpression had lower DFS and DMFS rates. These results are in agreement with the high correlation among PFDN subunits in the tumor tissue.

PFDN2 and 6 are also present in the unconventional prefoldin RPB5 interactor (URI)-prefoldin-like complex, which has been related to several types of cancer but not LC [12]. However, PFDN1, 3, and 5 are not present in the Uri-complex, showing that the detected clinical effects in LC are mediated by canonical PFDN, likely mediated by its nuclear role acting on cyclin A expression, as proposed by Wang et al. [8]. PFDN1 suppressed cyclin A expression by directly interacting with the cyclin A promoter at the transcriptional start site. Strikingly, cyclin A overexpression abolished the above PFDN1-mediated effects on the behavior of lung cancer cells, whereas cyclin A knockdown alone induced EMT and increased cell migration and invasion ability [8]. We conclude that overexpression of canonical PFDN is a prognostic marker of mortality and metastasis in NSCLC.

We acknowledge several limitations of our study. First, the small number of patients enrolled in the study was heterogeneous, having been treated over more than a decade, during which time advances in imaging and therapeutic regimens have occurred. Additionally, patients received different combinations of surgery \pm chemoradiation therapy rather than being treated according to a well-defined treatment protocol. To reduce the risk of bias, we analyzed the effect of PFDN overexpression in a more recent cohort and found similar results. Moreover, the type of surgery, radiotherapy, or chemotherapy among patients with and without PFDN1, 3, and 5 overexpression was assessed, and there were no significant differences. Currently, our group is expanding this study in a larger and more homogeneous cohort of patients recently treated. The influence of common lung cancer mutations (i.e., EGFR, ALK, etc.) or programmed cell death-ligand 1 (PD-L1) in the PFDN expression could be elucidated in this further study.

Poor genome-wide correlation between expression levels of mRNA and protein has been reported [13,14]. Lack of correlation between PFDN mRNA and PFDN protein levels, due to translational or protein turn-over regulation, may explain why PFDN was not included in any of the expression signatures so far proposed for the prognosis of LC based on transcriptomics (see Biswas et al. [15] and the other nine previous signatures cited therein). Other EMT protein markers with high prognostic significance in NSCLC, such as Snail and TWIST1 [16], were also out of published prognostic transcriptomic signatures. Our results indicate that protein response markers, such as PFDN1, 3, and 5, may complement mRNA signatures and be useful for determining the most appropriate therapy for NSCLC patients.

\section{Conclusions}

Our results show that overexpression of canonical PFDN associates with the risk of mortality and higher rates of recurrence, specifically for distant metastasis, in NSCLC. This response marker may complement mRNA signatures and be useful for guiding intensity in individualized therapy for NSCLC patients.

Supplementary Materials: The following are available online at http:/www.mdpi.com/2072-6694/12/4/1052/s1, Figure S1: Prefoldin expression was semi-quantitatively scored by two independent pathologists on a range of 0 to 3+, Figure S2: Kaplan-Meier curves of all 58 patients for disease-free survival according PFDN expression, Figure S3: Kaplan-Meier curves of all 58 patients for (A) disease-free survival (DFS) and (B) distant metastasis-free 
survival (DMFS) when combining all overexpressed PFDNs. Patients with PFDN1 and 3 overexpression had lower (A) DFS and (B) DMFS, Table S1: Univariate analyses of the associations between patient characteristics and PFDN protein expression levels and the outcome, Table S2: Multivariate analyses of the associations between patient characteristics and PFDN expression and outcome.

Author Contributions: Conceptualization, J.C.R., S.C. and J.L.L.G.; methodology, J.M.P.-F., J.C.R., S.C. and J.L.L.G.; software, J.M.P.-F., J.J.O., and J.C.R.; validation, X.P., J.M.P.-F., J.C.R., S.C. and J.L.L.G.; formal analysis, J.M.P.-F., J.J.O., and J.C.R.; investigation, X.P., P.R.P., and M.d.V.E.-R.; resources, J.M.P.-F., J.C.R., S.C. and J.L.L.G.; data curation, J.M.P.-F., P.R.P., M.d.V.E.-R., B.V., L.G.-I., J.J.O., and J.L.L.G.; writing-original draft preparation, X.P., E.R.d.C., J.C.R., S.C. and J.L.L.G.; writing-review and editing, X.P., J.M.P.-F., P.R.P., E.R.d.C., J.C.R., S.C. and J.L.L.G.; visualization, X.P., J.M.P.-F., P.R.P., M.d.V.E.-R., L.P.-B., B.V., L.G.-I., J.J.O., E.R.d.C., J.C.R., S.C. and J.L.L.G.; supervision, J.C.R., S.C. and J.L.L.G.; project administration, J.C.R., S.C. and J.L.L.G.; funding acquisition, J.C.R. and S.C. All authors have read and agreed to the published version of the manuscript.

Funding: This work was co-funded by the Spanish Ministry of Economy and Competitiveness (BFU2017-85420-R to JCR and BFU2016-7772-C3-1-P to SC) and by the Andalusian Government (BIO-271) and the University of Seville (US-1256285 to SC) in cooperation with the European Regional Development Fund.

Acknowledgments: The authors thank the donors and the Hospital Universitario Virgen del Rocío-Instituto de Biomedicina de Sevilla Biobank (Andalusian Public Health System Biobank and ISCIII-Red de Biobancos PT17/0015/0041) for the human specimens used in this study.

Conflicts of Interest: The authors declare no conflict of interest. The funders had no role in the design of the study; in the collection, analyses, or interpretation of data; in the writing of the manuscript, or in the decision to publish the results.

\section{References}

1. Mittal, V. Epithelial Mesenchymal Transition in Tumor Metastasis. Annu. Rev. Pathol. 2018, 13, $395-412$. [CrossRef] [PubMed]

2. Roche, J. The Epithelial-to-Mesenchymal Transition in Cancer. Cancers (Basel) 2018, 10, 52. [CrossRef] [PubMed]

3. Brabletz, T. To differentiate or not-routes towards metastasis. Nat. Rev. Cancer 2012, 12, 425-436. [CrossRef] [PubMed]

4. Gupta, P.B.; Pastushenko, I.; Skibinski, A.; Blanpain, C.; Kuperwasser, C. Phenotypic Plasticity: Driver of Cancer Initiation, Progression, and Therapy Resistance. Cell Stem Cell 2019, 24, 65-78. [CrossRef] [PubMed]

5. Lou, Y.; Diao, L.; Cuentas, E.R.; Denning, W.L.; Chen, L.; Fan, Y.H.; Byers, L.A.; Wang, J.; Papadimitrakopoulou, V.A.; Behrens, C.; et al. Epithelial-Mesenchymal Transition Is Associated with a Distinct Tumor Microenvironment Including Elevation of Inflammatory Signals and Multiple Immune Checkpoints in Lung Adenocarcinoma. Clin. Cancer Res. 2016, 22, 3630-3642. [CrossRef] [PubMed]

6. Lamouille, S.; Xu, J.; Derynck, R. Molecular mechanisms of epithelial-mesenchymal transition. Nat. Rev. Mol. Cell Biol. 2014, 15, 178-196. [CrossRef] [PubMed]

7. Lovisa, S.; LeBleu, V.S.; Tampe, B.; Sugimoto, H.; Vadnagara, K.; Carstens, J.L.; Wu, C.C.; Hagos, Y.; Burckhardt, B.C.; Pentcheva-Hoang, T.; et al. Epithelial-to-mesenchymal transition induces cell cycle arrest and parenchymal damage in renal fibrosis. Nat. Med. 2015, 21, 998-1009. [CrossRef] [PubMed]

8. Wang, D.; Shi, W.; Tang, Y.; Liu, Y.; He, K.; Hu, Y.; Li, J.; Yang, Y.; Song, J. Prefoldin 1 promotes EMT and lung cancer progression by suppressing cyclin A expression. Oncogene 2017, 36, 885-898. [CrossRef] [PubMed]

9. Vainberg, I.E.; Lewis, S.A.; Rommelaere, H.; Ampe, C.; Vandekerckhove, J.; Klein, H.L.; Cowan, N.J. Prefoldin, a chaperone that delivers unfolded proteins to cytosolic chaperonin. Cell 1998, 93, 863-873. [CrossRef]

10. Millan-Zambrano, G.; Chavez, S. Nuclear functions of prefoldin. Open Biol. 2014, 4, 140085. [CrossRef] [PubMed]

11. Payan-Bravo, L.; Penate, X.; Chavez, S. Functional Contributions of Prefoldin to Gene Expression. Adv. Exp. Med. Biol. 2018, 1106, 1-10. [PubMed]

12. Chaves-Perez, A.; Thompson, S.; Djouder, N. Roles and Functions of the Unconventional Prefoldin URI. Adv. Exp. Med. Biol. 2018, 1106, 95-108. [PubMed]

13. Abreu, R.d.; Penalva, L.O.; Marcotte, E.M.; Vogel, C. Global signatures of protein and mRNA expression levels. Mol. BioSyst. 2009, 5, 1512-1526.

14. Vogel, C.; Marcotte, E.M. Insights into the regulation of protein abundance from proteomic and transcriptomic analyses. Nat. Rev. Genet. 2012, 13, 227-232. [CrossRef] [PubMed] 
15. Biswas, D.; Birkbak, N.J.; Rosenthal, R.; Hiley, C.T.; Lim, E.L.; Papp, K.; Boeing, S.; Krzystanek, M.; Djureinovic, D.; la Fleur, L.; et al. A clonal expression biomarker associates with lung cancer mortality. Nat. Med. 2019, 25, 1540-1548. [CrossRef] [PubMed]

16. Hung, J.J.; Yang, M.H.; Hsu, H.S.; Hsu, W.H.; Liu, J.S.; Wu, K.J. Prognostic significance of hypoxia-inducible factor-1alpha, TWIST1 and Snail expression in resectable non-small cell lung cancer. Thorax 2009, 64, 1082-1089. [CrossRef] [PubMed]

C 2020 by the authors. Licensee MDPI, Basel, Switzerland. This article is an open access article distributed under the terms and conditions of the Creative Commons Attribution (CC BY) license (http://creativecommons.org/licenses/by/4.0/). 